\title{
Non-Dominated Sorting Flower Pollination Algorithm for Dynamic Economic Emission Dispatch
}

\author{
P. Paramasivan \\ Research Scholar \\ Department of ElectricalEngg, \\ Annamalai University, Annamalai nagar, \\ Tamilnadu, India
}

\author{
R.K. Santhi, PhD \\ Professor \\ Department of Electrical Engg, \\ Annamalai University, Annamalai nagar, \\ Tamilnadu, India
}

\begin{abstract}
This paper presents a non-dominated sorting flower pollination algorithm for dynamic economic emission dispatch (DEED) problem. Non-dominated sorting flower pollination algorithm is designed to construct the pareto optimal front and a fuzzy techniques extracts the best compromised solution of DEED. Results two standard of test systems are presented to exhibit its superior performance.
\end{abstract}

\section{Keywords}

Pareto Optimal Front, Predator Prey Optimization Flower Pollination Algorithm

\section{INTRODUCTION}

Optimal scheduling of thermal units, one of the most important functions in power system operation and planning, can lead to significant cost savings. The traditional solution techniques encounter difficulties such as getting trapped at local optima and increased computational complexity. Scarcity in energy resources, increasing power generation cost, ever-growing load demand and increasing concern over the environmental considerations for electric energy necessitate developing better DEED solution techniques. The method of combing multiobjectives into single objective problem through weight approach may not give best compromised solution (BCS). However, how much attention should be paid to each objective is often based on a conceptual understanding of the problem. In many situations, there is no mathematical approach that can precisely quantify those conceptual objectives into numerical weight coefficients. Besides it requires several solution runs with different $w$ values for constructing the Pareto Front with a view of obtaining the BCS, which can be defined as the one having equal percent deviations from the best values of the individual objective functions besides lying nearer to the best solutions of the individual objectives. The aim of this paper is to develop a non-dominated sorting Self Adaptive Predator Prey Flower Pollination Algorithm (SAPPFPA) strategy for biobjective Dynamic Economic Emission Dispatch (DEED) problem for obtaining the Pareto optimal front in a single solution run and then extracting the BCSs from the Pareto optimal front (POF) using fuzzy technique.

\section{PROBLEM FORMULATION}

The problem of Dynamic Economic Emission Dispatch (DEED) involves simultaneous optimization of the following two incomparable and conflicting objectives, leading to a set of non-dominated solutions known as Pareto-optimal set (POS).

Minimize $\Phi_{F E}\left(P_{G}\right)=\left[f_{1}(x), f_{2}(x)\right]$

Subject to

$$
\begin{aligned}
& \sum_{i=1}^{n g} P_{G i t}-P_{D t}-P_{L t}=0 \quad t \in n t \\
& P_{G i}^{\min } \leq P_{G i t} \leq P_{G i}^{\max } \quad i \in n_{g}, t \in n t \\
& P_{G i t}-P_{G i t-1} \leq U R_{i} \quad i \in n_{g} \quad t=2, \cdots, n t \\
& P_{G i t-1}-P_{G i t} \leq D R_{i} \quad i \in n_{g} \quad t=2, \cdots, n t
\end{aligned}
$$

Where

$$
\begin{aligned}
& f_{1}(x)=\sum_{t=1}^{n t} \sum_{i=1}^{n g} F_{i}\left(P_{G i t}\right) \\
& f_{2}(x)=\sum_{t=1}^{n t} \sum_{i=1}^{n g} E_{i}\left(P_{G i t}\right) \\
& F_{i}\left(P_{G i t}\right)=a_{i} P_{G i t}^{2}+b_{i} P_{G i t}+c_{i}+\left|d_{i} \sin \left\{e_{i}\left(P_{G i}^{\mathrm{min}}-P_{G i t}\right)\right\}\right| \\
& E_{i}\left(P_{G i}\right)=\alpha_{i} P_{G i}{ }^{2}+\beta_{i} P_{G i}+\gamma_{i}+\xi_{i} \exp \left(\delta_{i} P_{G i}\right) \\
& p_{L, t}=\sum_{j=1}^{N} \sum_{j=1}^{N} p_{j, t} B_{G i t} p_{j, t}+\sum_{j=1}^{N} B_{o, j} P_{j, t}+B_{o, o}
\end{aligned}
$$

\section{FLOWER POLLINATION ALGORITHM}

The Flower Pollination Algorithm (FPA) is inspired by the pollination process of flowering plants. The objective of flower pollination is the survival of the fittest and the optimal reproduction of plants in terms of numbers as well as the fittest. FPA has been adopted through the following four steps:

Step 1: Global pollination represented in biotic and crosspollination processes, as pollen carrying pollinators fly following Lévy flight .

Step 2: Local pollination represented in abiotic and selfpollination as the process does not require any pollinators.

Step 3: Flower constancy which can be developed by insects, which is on a par with a reproduction probability that is proportional to the similarity of two flowers involved.

Step 4: The interaction of local and global pollination is controlled by a switch 
Probability $p \in[0,1]$, lightly biased toward local pollination.

we also assume that each plant only has one flower, and each flower only produce one pollen gamete for simplicity. This simplicity means a solution $x_{i}$ is equivalent to a flower and/or a pollen gamete. There are two key steps in this algorithm; they are global pollination and local pollination. In the global pollination step, flower pollens are carried by pollinators such as insects, and pollens can travel over a long distance because insects can often fly and move in a much longer range and can be represented by:

$$
x_{l}^{t+1}=x_{l}^{t}+\gamma L(\lambda)\left(x_{l}^{t}-g^{*}\right)
$$

where $x_{l}^{t}$ is the pollen of flower- $l$ at $t$-th iteration and $g *$ is the current best solution found among all solutions at the current generation. Here $\gamma$ is a scaling factor controlling the step size. $L(\lambda)$ is the Lévy flights that represents the strength of the pollination.

$$
L \approx \frac{\lambda \Gamma(\lambda) \sin (\pi \lambda / 2)}{\pi} \frac{1}{s^{1+\lambda}}, \quad\left(s>>s_{o}>0\right)
$$

Here $\Gamma(\lambda)$ is the standard gamma function, and this distribution is valid for large steps $s>0$. For the local pollination, both Step 2 and Step 3 can be represented as

$x_{l}^{t+1}=x_{l}^{t}+\varepsilon\left(x_{n}^{t}-x_{p}^{t}\right)$

where $x_{n}^{t}$ and $x_{p}^{t}$ are pollen from different flowers of the same plant species mimicking the flower constancy in a limited neighborhood. For a local random walk, $x_{n}^{t}$ and $x_{p}^{t}$ comes from the same species then $\varepsilon$ is drawn from a uniform distribution as $[0,1]$.

The FPA steps are outlined below:

1. Choose the parameters population size, maximum number of iterations for termination, switch probability $p$, etc

2. Initialize a population of $N F$ flowers with random solutions.

3. Evaluate the objective function of each flower in the population.

4. Find/Update the current best solution.

5. For each flower in the population, generate a random number in a range of $(0,1)$. If this random number is less than the $p$, perform local pollination, else do global pollination using Levy flight.

6. Outputs the best solution, if the termination criteria are met, else go to step (3).

7. Stop.

\subsection{Adaptive Mutation}

The adaptive mutation rate can be heuristically evaluated from the diversity measure $D(t)$ of the population at generation- $t$ as

$P_{m}(t+1)=P_{m}^{0} \cdot\left(1+\frac{D(1)-D(t)}{D(1)}\right)$

where

$D(t)=\frac{1}{n f \cdot \chi} \cdot \sum_{i=1}^{n f} \sqrt{\sum_{j=1}^{n d}\left(x_{i j}-\bar{x}_{j}\right)^{2}}$

$\bar{x}_{j}=\frac{1}{n f} \cdot \sum_{i=1}^{n f} x_{i j}$

\subsection{Predator-prey model}

The property of predators helps the preys to explore search area more effectively. The predators are modeled based on the worst solutions as

$$
x_{\text {predator }}(t)=x_{\text {worst }}(t)+\rho \cdot\left(1-\frac{t}{t_{\max }}\right)
$$

The fugue of prey can be modeled through maintaining distance between predator and prey as

$$
\begin{aligned}
& x(t+1)=x(t)+\rho \cdot e^{-|d|}, \quad \text { if } d>0 \\
& x(t+1)=x(t)-\rho \cdot e^{-|d|}, \quad \text { if } d<0
\end{aligned}
$$

The algorithmic steps of the APPFPA are outlined below:

1. Choose the parameters population size, maximum number of iterations for termination, switch probability $p$, etc

2. Initialize a population of $N F$ flowers with random solutions.

3. Evaluate the objective function of each flower in the population.

4. Find/Update the current best solution.

5. Evaluate the population diversity using Eq. (14).

6. Compute mutation rate using Eq. (13)

7. Perform the following for each flower in the population

a. Generate a random number in a range of $(0,1)$. If this random number is less than the $p$, perform local pollination, else do global pollination using Levy flight.

b. Probabilistically hunt the solutions using Eqs. (16) and (17)

c. Probabilistically perform mutation based on the mutation rate

8. Output the best solution, if the termination criteria is met, else go to step (3)

9. Stop.

\section{NON DOMINATED SORTING}

The reason for optimality of many solutions is that no one can be considered to be better than any other with respective to all 
objective functions of POS. The Pareto Optimal Set (POS) involving SAPPFPA is tailored to obtain the POS in single solution run. The suggested method involves the representation of design variables as depicted in Fig.1, a procedure for choosing the non-dominated solutions in the population and a process of computing fuzzy membership function.

A solution belongs to the POS, if there is no other solution that can improve at least one of the objectives without degrading any other objective. Any two different feasible solutions $x_{1}$ and $x_{2}$ of any multi-objective problem can have one of two possibilities: one dominates the other or none dominates the other. $x_{1}$ Is called the non-dominated solution, when $x_{1}$ dominates $x_{2}$ through satisfying the following two conditions:

$$
\begin{aligned}
& \forall i \in\left\{1,2, \cdots, n_{\text {obj }}\right\}, \quad f_{i}\left(x_{1}\right) \leq f_{i}\left(x_{2}\right) \\
& \exists j \in\left\{1,2, \cdots, n_{\text {obj }}\right\}, \quad f_{j}\left(x_{1}\right)<f_{j}\left(x_{2}\right)
\end{aligned}
$$

Where,

$n_{o b j}$ is the number of objective functions.

The Pareto Optimal Front (POF) is obtained by mapping these non-dominated solutions into the objective space as follows:

$$
P O F=\left\{f_{1}(x), f_{2}(x), f_{3}(x), \cdots, f_{n_{o b j}}(x) \mid x \in \Phi\right\}
$$

Where,

$\Phi$ is the set of non-dominated solutions obtained by the algorithm.

An external archive is used in the proposed method Pareto Optimal Set (POS) to save the non-dominated solutions during the iterative process. The non-dominated solutions, obtained at the end of each iteration, are added in the archive and nondominance checking is performed for both the new members and the old members of the archive. The non-dominated members of the archive will remain in the archive and other dominated members will be removed.

The obtained POS may be enormously large and requires reducing the set of non-dominated solutions without destroying the characteristics of the POF. A truncation algorithm that works iteratively is suggested to reduce the POS to the required archive size. It uses the measure of Euclidean distance between two members of the archive and membership value of each member. The Euclidean distance in the objective space is calculated using

$d_{i j}=\sqrt{\sum_{k=1}^{n_{o b j}}\left(\frac{f_{k}^{i}-f_{k}^{j}}{f_{k}^{U}-f_{k}^{L}}\right)^{2}}$

Where

$d_{i j}$ Is the Euclidean distance between two members- $i$ and $j$ of the archive? $f_{k}^{i}$ Is the value of the $k^{t h}$ objective of $i^{t h}$ member of the archive?

$f_{k}^{L}$ And $f_{k}^{U}$ represent the lower and upper value of the $k^{t h}$ objective of the archive.

$n_{\text {obj }}$ Indicates the number of objectives in the problem.

The fuzzy membership of $i^{t h}$ member can be calculated by

$\mu^{i}=\frac{\sum_{k=1}^{n_{o b j}} \mu_{k}^{i}}{\sum_{i=1}^{n_{n d s}} \sum_{k=1}^{n_{o b j}} \mu_{k}^{i}}$

$n_{n d s} \quad$ Number of non-dominated solutions

Where $\mu_{k}^{i}$ represents the membership of the $k^{t h}$ objective of $i^{t h}$ member of the archive and can be calculated by

$\mu_{k}^{i}=\left\{\begin{array}{cc}1 & f_{k}^{i} \leq f_{k}^{L} \\ \frac{f_{k}^{U}-f_{k}^{i}}{f_{k}^{U}-f_{k}^{L}} & f_{k}^{L}<f_{k}^{i}<f_{k}^{U} \\ 0 & f_{k}^{i} \geq f_{k}^{U}\end{array}\right.$

The truncation algorithm may be explained with a given a nondominated solution set $\Phi$ whose size $n_{n d s}$ exceeds the maximum archive size $n_{\max }$ through the following steps.

While $n_{\text {nds }}>n_{\max }$, do the following

- Calculate the Euclidean between two members, $i$ and $\mathrm{j}$, of the archive using Eq. (18)

- Choose the pair with lowest distance and calculate their membership using Eq.(19)

- $\quad$ Remove the member with lower membership

End

\section{PROPOSED METHOD}

The proposed method involves representation of problem variables and formation of a fitness function. Considering the $P_{G}$ as the problem variables, the pollen of each flower can be represented by a real number matrix as shown in Fig.1. The variable $P_{G i t}$ represents real power generation of unit- $i$ at interval- $t$. 


\begin{tabular}{l|c|c|c|c|c|c|}
\multicolumn{2}{c}{1} & \multicolumn{2}{c}{2} & \multicolumn{2}{c|}{} \\
\cline { 2 - 7 } 1 & $P_{G 1,1}$ & $P_{G 1,2}$ & $P_{G 1,3}$ & $P_{G 1,4}$ & $\cdots$ & $P_{G 1, n i}$ \\
\cline { 2 - 7 } & $P_{G 2,1}$ & $P_{G 2,2}$ & $P_{G 2,3}$ & $P_{G 2,4}$ & $\cdots$ & $P_{G 2, n i}$ \\
\hline & $\vdots$ & & $\vdots$ & & $\vdots$ \\
\hline & $P_{G n g, 1}$ & $P_{G n g, 2}$ & $P_{G n g, 3}$ & $P_{G n g, 4}$ & $\cdots$ & $P_{G n g, n i}$ \\
\hline
\end{tabular}

Fig.1 Representation of Pollen of Each Flower

The fitness function of can be built from fuzzy membership function of Eq. (22) and the penalty terms representing the limit violations of explicit constraints of Equation as.

$$
F_{i}=\mu^{i}+k_{3}\left(\frac{1}{1+\sum_{t=1}^{n i}\left\{\sum_{i=1}^{n g} P_{G i t}-P_{D t}-P_{L t}\right\}^{2}}\right)
$$

An empty archive is defined and an initial population of students containing the grade points is obtained by generating random values within their respective limits. The nondominated solutions are identified in the population and the archive is updated through storing the non-dominated solutions of the population. The truncation algorithm is applied, if the size of the archive exceeds the maximum allowable limit. The fuzzy membership $\mu^{i}$ is calculated by considering pollens of each flower in the population and as well in the archive. The member possessing largest fuzzy membership in the archive is considered as the global best flower and the one inheriting smallest membership in the population is taken as the predator. The global pollination, local pollination, hunting and mutation phases are performed for all the flowers in the population with a view of minimizing their cost functions. The above iterative process is continued till convergence. The set of nondominated solutions in the archive represents the POS obtained by the proposed approach. The member with highest fuzzy membership in the archive is taken as the BCS. The flow chart of the proposed non-dominated sorting APPFPA in POS for BCS of DEED is explained in Fig. 2. 


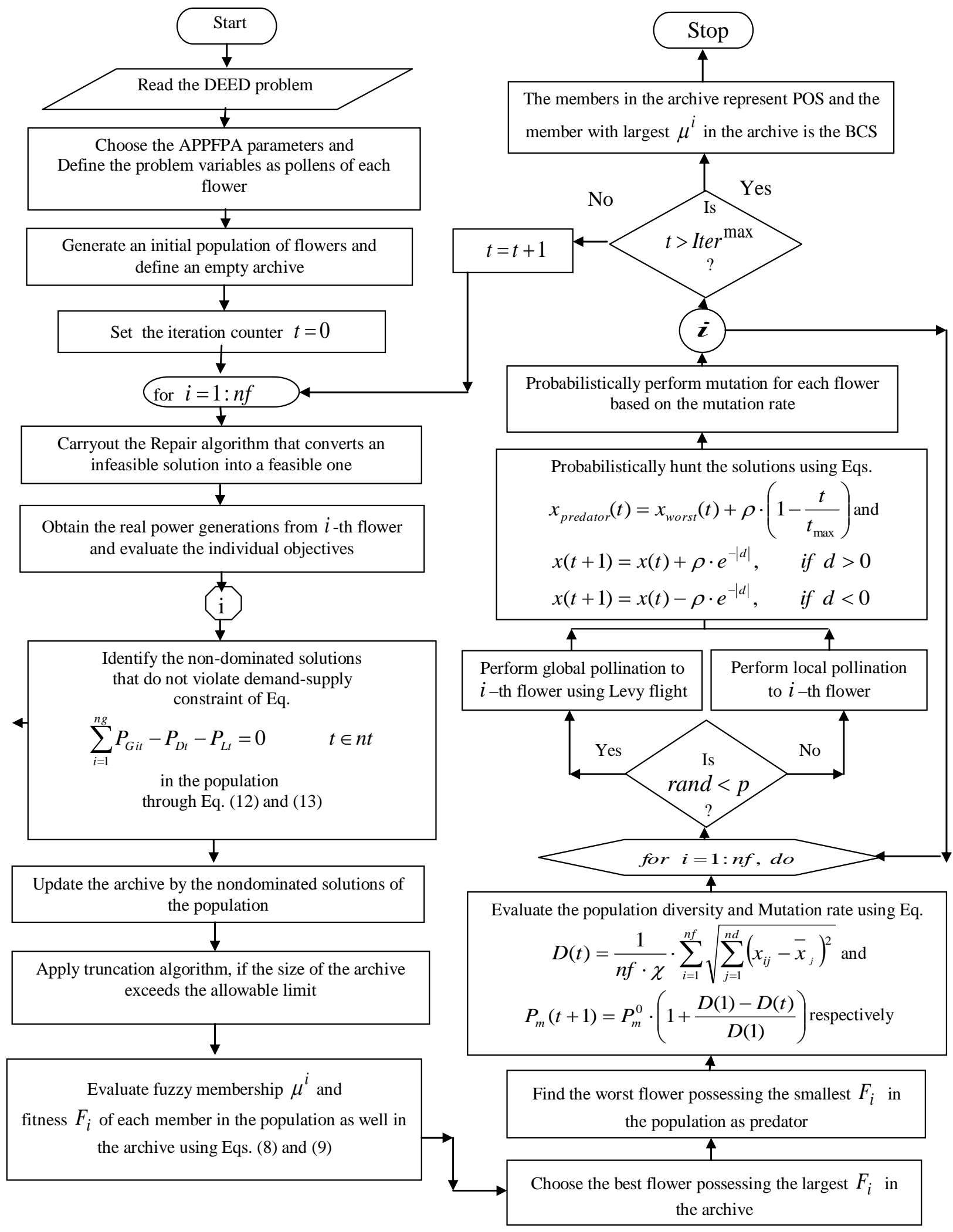

Fig.2. Flow chart of the Proposed Method 


\section{SIMULATIONS}

The proposed non-dominated sorting APPFPA method POS for BCS is applied on two different test cases with varying degree of complexity for studying its performance. The first one possesses 5 generating [19] units, the second system comprises 10 generators [19]. Besides the results of the proposed method are compared with that of the weighting approach. The software packages are developed in Matlab platform and executed in a $2.3 \mathrm{GHz}$ Pentium-IV personal computer. There is no guarantee that different executions of the developed design programs converge to the same design due to the stochastic nature of the FFO and ACO, and hence the algorithms are run 20 times for each test case and the best ones are presented in this section.

The normalized objective function value (NOV) that represents how far the solution is away from the individual best points, is used for studying the goodness of the solution.

$$
\text { NOV } i=\left(\frac{f_{i}-f_{i}^{\text {Best }}}{f_{i}^{\text {Worst }}-f_{i}^{\text {Best }}}\right) \times 100
$$

Table 1. Best and worst objective function values

\begin{tabular}{|c|c|c|c|c|}
\hline \multirow{2}{*}{ Test } & \multicolumn{2}{|c|}{ FC } & \multicolumn{2}{c|}{ EC } \\
\cline { 2 - 5 } Case & Best & Worst & Best & Worst \\
\hline 1 & 43047.96 & 52085.91 & 17866.53 & 23482.45 \\
\hline 2 & $2.4659 \times 10^{6}$ & $2.5905 \times 10^{6}$ & $2.9196 \times 10^{6}$ & $3.2405 \times 10^{6}$ \\
\hline
\end{tabular}

A solution is said to be BCS if the sum of NOVs (SNOV) is the lowest besides the NOV of both the objectives are in the same range, which can be assessed through calculating the difference between the minimum and maximum NOV values (DNOV) of the chosen objectives. The best and worst values of the individual objectives, required for evaluating the NOVS for all the test cases are given in Table 2 .

The BCS representing the real power generations of the SAPPFPA for all the test cases are graphically represented in Fig. 3-6

Table 2.Comparison of Results of POS for Test Case- 1

\begin{tabular}{|c|c|c|c|c|}
\hline & $\mathrm{W}=0.5$ & $\mathrm{~W}=0.5$ & NOFV-1 & NOFV-2 \\
\hline & $\begin{array}{c}\text { Fuel Cost } \\
{[\$ / h]}\end{array}$ & $\begin{array}{c}\text { Emission } \\
{[\mathrm{kg} / \mathrm{h}]}\end{array}$ & & \\
\hline $\begin{array}{c}\text { Weight } \\
\text { method }\end{array}$ & 46195.74 & 18542.03 & 34.83 & 12.03 \\
\hline PM & 44910.97 & 18954.91 & 20.61 & 19.38 \\
\hline
\end{tabular}

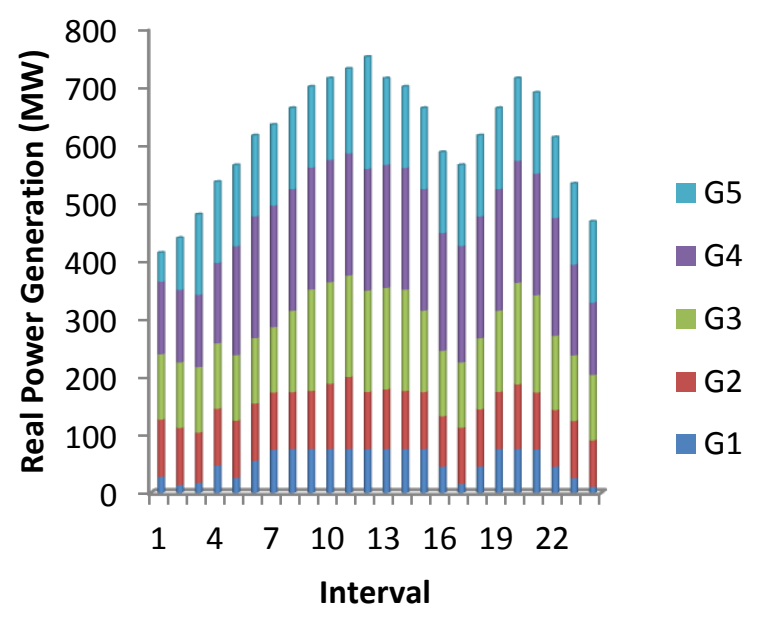

Fig. 3.Real Power Generations of PM for Test Case-1

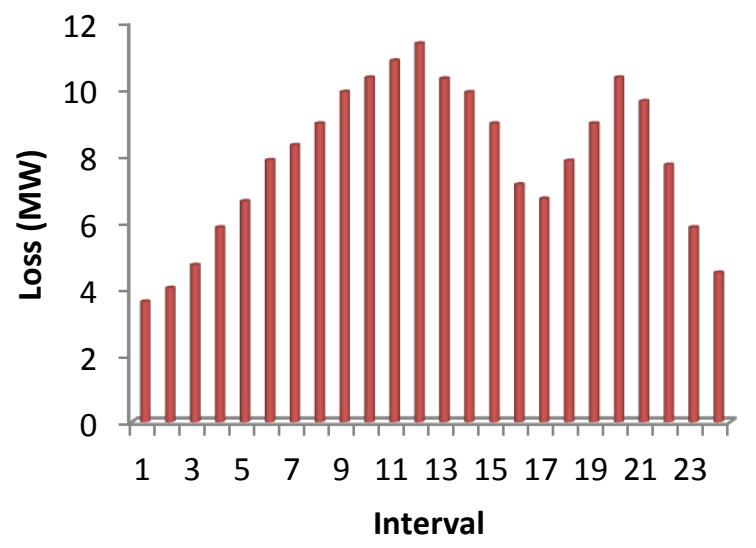

Fig. 4. Network Loss at each interval of PM for Test Case1

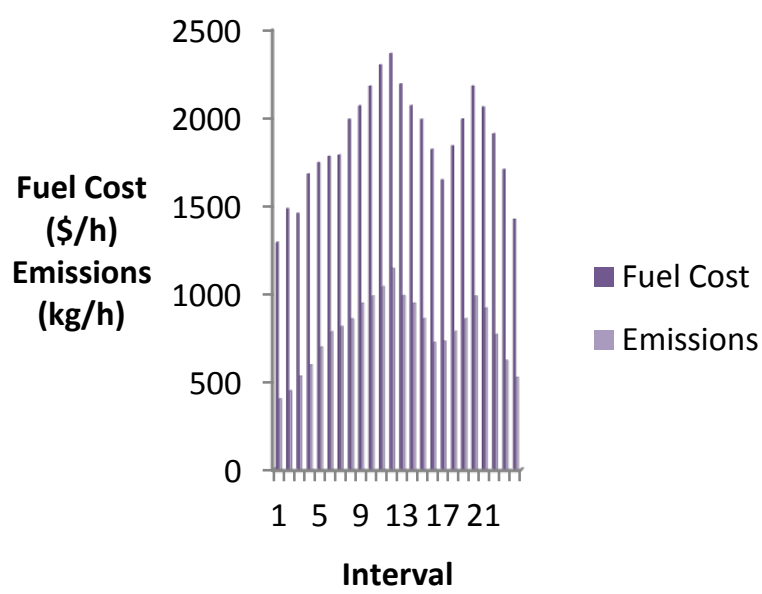

Fig. 5. Fuel Cost and Emissions at each interval by PM for Test Case-1 
Table 3.Comparison of Results of PM for Test Case-2

\begin{tabular}{|c|c|c|c|c|}
\hline & DEED & & $\begin{array}{c}\text { NOFV- } \\
\mathbf{1}\end{array}$ & $\begin{array}{c}\text { NOFV- } \\
\mathbf{2}\end{array}$ \\
\hline & $\begin{array}{c}\text { Fuel Cost } \times \\
\mathbf{1 0}^{6}[\$ / h]\end{array}$ & $\begin{array}{c}\text { Emissions } \times \\
\mathbf{1 0}^{5} \\
{[\mathrm{~kg} / \mathrm{h}]}\end{array}$ & & \\
\hline $\begin{array}{c}\text { Weight } \\
\text { method }\end{array}$ & 2.510020167 & 3.011571061 & 35.3918 & 28.6510 \\
\hline PM & 2.506596316 & 3.019168144 & 32.6446 & 31.0184 \\
\hline
\end{tabular}

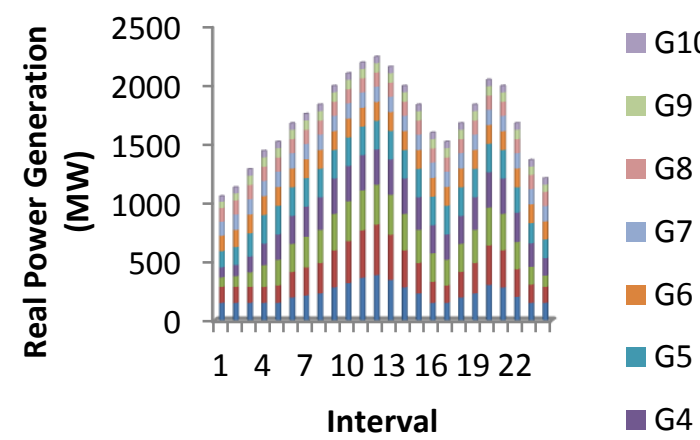

Fig. 6 Real Power Generations of PM for Test Case-2

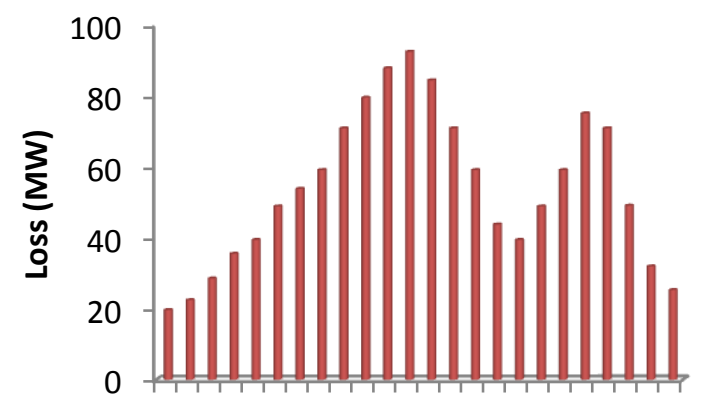

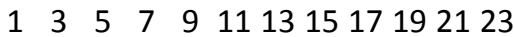

Interval

Fig. 7 Network Loss at each interval of PM for Test Case-2

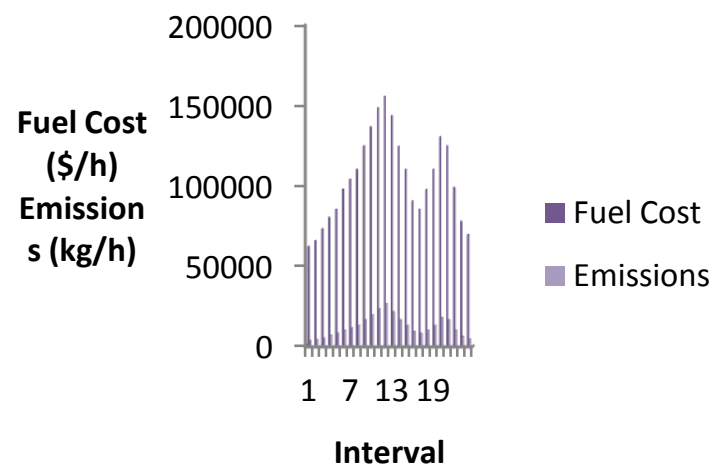

Fig. 8 Fuel Cost and Emissions at each interval by PM for Test Case-2

\section{CONCLUSION}

The non-dominated sorting concept has been blended with APPFPA and applied on DEED with a view of obtaining the $\mathrm{BCS}$ that exactly gives equal significance to both the fuel cost and emissions. The adaptive mutation helps to explore newer regions in the search space and the predator-prey concept prevents local minima. The developed algorithm is able to after the global best solution. The results on two test system have clearly indicates the superior performance of the algorithm.

\section{ACKNOWLEDGMENT}

The authors gratefully acknowledge the authorities of Annamalai University for their continued support, encouragement, and the extensive facilities provided to carry out this research work.

\section{REFERENCES}

[1] M.Basu, "Dynamic economic emission dispatch using non-dominated sorting genetic algorithm-II," Electrical power and Energy systems 30 (2008) 140-149.

[2] Li xuebin , "Study of multi-objective optimization and multi-attribute decision-making for dynamic economic emission dispatch," Electric power components and systems, vol. 37: pp 1133-1148, 2009.

[3] Elaiw A.M., Xia X,.Shehata A.M, "Hydrid DE-SQP and hybrid PSO-SQP methods for solving dynamic economic emission dispatch problem with valve point effects," Electric Power Systems Research 103 (2013) 192-200.

[4] M. Basu, " Artificial Immune System for dynamic economic dispatch," Electrical power and energy systems 33 (2011) 131-136.

[5] Pandi N, Tripati A, Tapaswi S padit M, "An improved bacterial foraging algorithm for combined static/dynamic environmental economic dispatch," Applied soft computing 2012; 12 (11) :3500-3513.

[6] Yong Zhang, Dun-wei Gong, NaGeng, Xiao-yan Sun, "Hybrid bare-bones PSO for dynamic economic dispatch with valve-point effects", Applied soft Computing 18 (2014) 248-260.

[7] R.Arul, S.Velusami, G.Ravi, "A new algorithm for combined dynamic economic emission dispatch with security constraints," Energy 79 (2015) 496-511.

[8] Nnamdi I, Nwulu, Xiaohua Xia, "Multi-objective dynamic economic emission dispatch of electric power generation integrated with game theory based demand response programs," Energy conservation and management 89 (2015) 963-974.

[9] Elaiw AM, Xia X, Shehata AM. Minimization of fuel costs and gaseous emissions of electric power generation by model predictive control. Math Prob Eng 2013;2013. Article ID 906958.

[10] Ramanathan R. Emission constrained economic dispatch. IEEE Trans Power Syst 1994;9:1994-2000.

[11] Hemamalini S, S.Simon, "Dynamic economic dispatch using artificial bee colony for units with valve point effect," European transactions on electrical power 21(2011) 70-81.

[12] El.keib AA, Ma H, Hart JL. Economic dispatch in view of the clean air act of 1990. IEEE Trans power system 
1994; 9 (2) : 972-978.

[13] IEEE current operating problems working group, "potential impacts of clean air regulations on system operations," IEEE trans power system. Vol.10, pp.647653,1995 .

[14] J.H.Talaq, F.El.Hawary, M.E.El. Hawary, "A summary of environmental/ economic dispatch algorithms, IEEE Transactions on power systems, 9 (1994) 1508-1516.

[15] M. Basu, “ Particle swarm optimization based goalattainment method for dynamic economic emission dispatch," Electric Power Components and Systems.34 (2006):1015-1025.

[16] J.S. Alsumait, M. Qasem, J.K. Sykulski, A.K. AlOthman.(2010). An improved pattern search based algorithm to solve the dynamic economic dispatch problem with valve-point effect, Energy Conversion and Management 51: 2062-2067.

[17] Xingwen jiang, jianzhong zhou, Hao wang, yongchuan zhang. Dynamic environmental economic dispatch using multiobjective differential evolution algorithm with expanded double selection and adaptive random restart. Electrical power \& Energy systems. (2013) 49:399-407.
[18] Panigrahi CK, Chattopadhyay PK, Chakrabarti RN, Basu.M. Simulated annealing technique for dynamic economic dispatch. Electr Power Compon Syst; (2006) 34:577-86.

[19] Basu M. Dynamic economic emission dispatch using evolutionary programming and fuzzy satisfying method. Int J Emerg Electr Power Syst 2007; 8 (art 1).

[20] B. Panigrahi, P.V. Ravikumar, D. Sanjoy, Adaptive particle swarm optimization approach for static and dynamic economic load dispatch, Energy Conversion and Management (2008) $49: 1407-1415$.

[21] V.R. Pandi, B.K. Panigrahi,. Dynamic economic load dispatch using hybrid swarm intelligence based harmony search algorithm, Expert Systems with Applications (2011) 38:8509-8514.

[22] R. Balamurugan, S. Subramanian.. An improved differential evolution based dynamic economic dispatch with non-smooth fuel cost function, Journal of Electrical Systems (2007) 3 (3): 151-161.

[23] Hemamalini S, Simon SP. Dynamic economic dispatch using artificial immune system for units with valve-point effect. Int J Electr Power Energy System. 2011;33:86874. 